\title{
The Design and Implementation of Network Video Surveillance System based on ASP.NET
}

\author{
Hongjun Jin \\ Yancheng Teachers University, Yancheng Jiangsu 224002, China \\ Jinhong@21cn.com
}

\begin{abstract}
Keywords: Software engineering, network video surveillance system, ASP.NET, B/S infrastructure, design pattern.
\end{abstract}

\begin{abstract}
In this paper, the author describe the application and developing trend of the video surveillance system , analyze and compare the different frameworks, make out the design of Network Video Surveillance based on ASP.NET Framework. Then give the design and implementation of media server module, alarm server module, user management module, equipment management module, log management module and electric map module. Finally, test the whole Video Surveillance System, and expect for the trend of the Network Video Surveillance System.
\end{abstract}

\section{Introduction}

Along with the development of network video surveillance system, developing a convenient and efficient network video surveillance system has become a hotspot for related network companies and software engineers in recent years. The advantages of ASPASP.NET include universal application, simple programming interface, low programming workload and compatible programming language. [1] So ASPASP.NET has become the first choice for related software engineers when developing a network system.

With the development of network communication technology and multimedia technology, video surveillance technology develops quickly. [2] But, without unified standard, the front equipments come from different manufacturers which apply with monitor centre software itself, and the system works independence. While more and more surveillance manufacturers switch in, the operation and the maintenance work of the system are getting more and more difficult. Be aimed at current situation, this paper presents a design of Network Video Surveillance System based on ASP.NET Framework, which realize distributed surveillance, centralized management, highly compatible and integrated with surveillance equipments of different manufacturers.

The video surveillance industry is a sunrise industry and the video monitoring system is an important part of the video surveillance industry. Appeared in the early 1970s, with the development of HD cameras, hard disk recorders, network cameras, and the progress in network technology, video codec technology, and video analysis technology, the video monitoring system has been refined [3]. The development of the video monitoring system has experienced three stages: analog monitoring systems, digital monitoring systems and network monitoring systems. It is developing into an integrated, network-based, intelligent system. The video monitoring system has broad application respects in security as well as the supervision and resource management in businesses, kindergartens, and financial industry.

\section{Network video surveillance system design}

Video surveillance system architecture: the architecture of the video surveillance system description and analysis of a video surveillance system in the central platform consisting of four levels, each level is introduced. Information storage technology: We introduce a centralized information storage technology, DAS, NAS, SAN and discs, the specific contents of these storage protocols, their strengths and weaknesses, is a microcosm of the development of storage technology. 
Video surveillance system construction: the development of video surveillance systems in this article is based on DirectShow technology is based on windows operating platform, system design and implementation, the system achieve up to 16-way video models while monitoring, and can automatically switch the lens paper log system to build a video surveillance system, run clear and complete record system, the use of the user's convenience for normal use and maintenance of the system. [4] Factory method diagram of video surveillance is shown in Fig. 1.

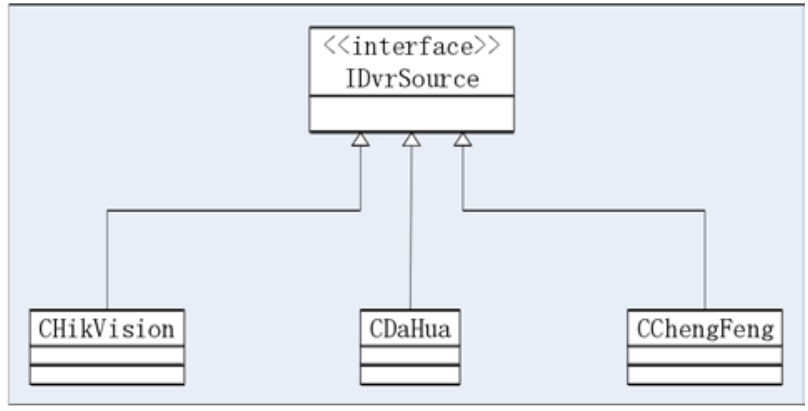

Fig. 1 Factory method diagram of video surveillance

At present, many industries have built many small-scale video monitoring networks. They may be analog video monitoring system composed of the analog cameras, QUAD System, video matrix, 丁V wall, or digital video monitoring system consisting of DVR, PC, video monitoring software provided by equipment manufacturers. Along with the gradually enrich of applications based on the video monitoring system, the future direction of video surveillance industry is the development of a province-wide network video monitoring platform witch is compatible with most vendors' equipments and integrated of existing digital and analog video resources.

In view of the various shortcomings to the traditional analog video surveillance system, through the method of analyzing and researching the embedded chip ASP.NET, the design of an embedded network video surveillance system based on ASP.NET chip is put forward [5]. The paper design and implement the real-time video on demand capability of the system in accordance with the typical software development process (requirements analysis, design, implementation, testing and software delivery), using the design principles of streaming media system. On the side of video server, the paper completes the video acquisition using Video for Linux interface, video encoding using the ASP.NET codec module within ASP.NET, exchange of control information with client using RTSP, transmission of media data using RTP. On the side of client, the paper uses DirectShow technology to complete the video decode and play. E.g. Factory method design pattern is shown in Fig. 2.

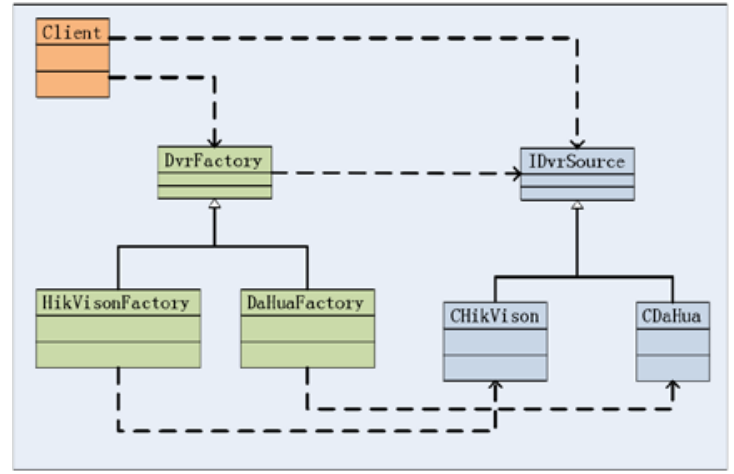

Fig. 2 Factory method design pattern

\section{Implementation of modular}

This thesis described the design and development of an application platform based on video monitoring system and ASP network. It supports accesses of digital coding equipment and analog matrix devices provided by most of the monitoring industry manufacturers. The platform consists of management server, interface server, transformation server, web server and client. The interface server and transformation server are implemented with data pool, thread pool, asynchronous I/O to achieve high performance servers, so as to meet the requirements of large numbers of client access 
and large amounts of data transformation. The web server uses the J2EE architecture with the capability of database configuration management. The client was developed based on OCX component technology, so clients can use the platform through the browser. The platform has many functional modules, such as real-time preview of the video, polling preview of the video, local video storage, local video query and playback, device video query and playback, PTZ control and so on. ASP.NET application access framework is shown in Fig. 3.

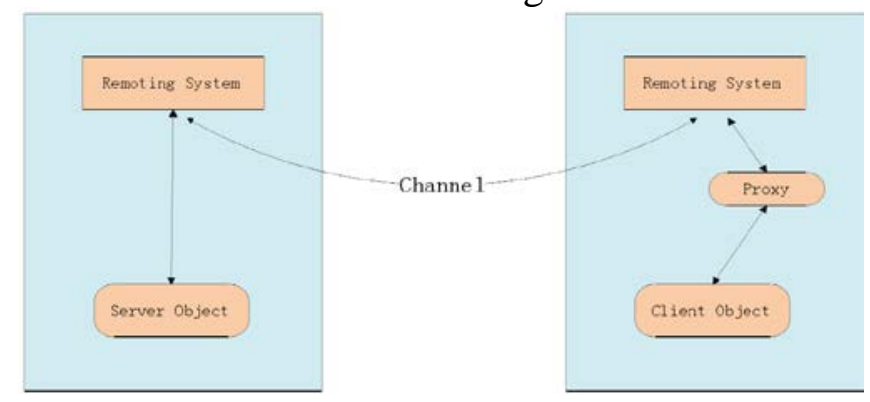

Fig. 3 ASP.NET application access framework

The exploitation of ASP.NET system is based on ASP.NET hardware platform and ASP.NET operating system. In this area, ASP.NET has becoming the mainstream operating system. ASP.NET which can be applied on many kinds of platform is open source and free. Itself can support strong network function and possess numerous software resources. It is the development of ASP.NET that make it possible to employer IPCAM, a high capability and low cost ASP.NET equipment.

The main research of this thesis is about the design and implementation of network video monitoring system based on the ASP.NET system on ARM9 platform. Focus on the video capture, transmission and client GUI design. Using B/S structure with special client software in system design, separating the ASP.NET video capture terminal function module and PC-client monitoring software, using RTP/RTCP Real-time Transport Protocol transmit the video streaming on network. In the ASP.NET video capture terminal, the USB camera driving programs based on video for Linux and video for Linux two is researched, and the image capturing by the two interface specification is realized. The USB driving program based on video for Linux is used in finial system. Taking into account the constraints of the hardware platform itself and reduce costs, each frame is compressed into a JPEG image format. The users can operate the monitoring system by the Web page through the establishment of the ASP.NET Web server. Client video surveillance software is developed by Qt in the Linux operating system. The software enables multiple real-time monitoring of the data source and provides

\section{Test and application}

The paper comes from the actual development projects of Beijing Top shine Technology Co., Ltd: wireless-board video surveillance system. As an integral part of Security Defense system, video surveillance system is seeing a rapid development with the technology innovation of ASP.NET system $\}$ multimedia and computer network. The current ASP.NET-system based network video surveillance system has a great prospect due to its better portability, lower cost, higher stability and better real-time feature, compared with the traditional video surveillance system. According to the requirement analysis, the paper has built a simulate test environment based on the $3 G$ network and test the system using a combination of black box testing and white box testing. The main content of the test is real-time video on demand capability. The testing performance achieved the desired design goals. In this thesis a detailed exposition of the key technical issues in the development of the video monitoring application platform is involved, including: the requirement analysis and design principles of the video monitoring application platform, the architecture of the video monitoring application platform, the main business processes in this video monitoring application platform and the implementation of it. This thesis writes based on practice and introduces the video monitoring application platform in detail, including the module structure, system processes, system interface, and device network solutions. 


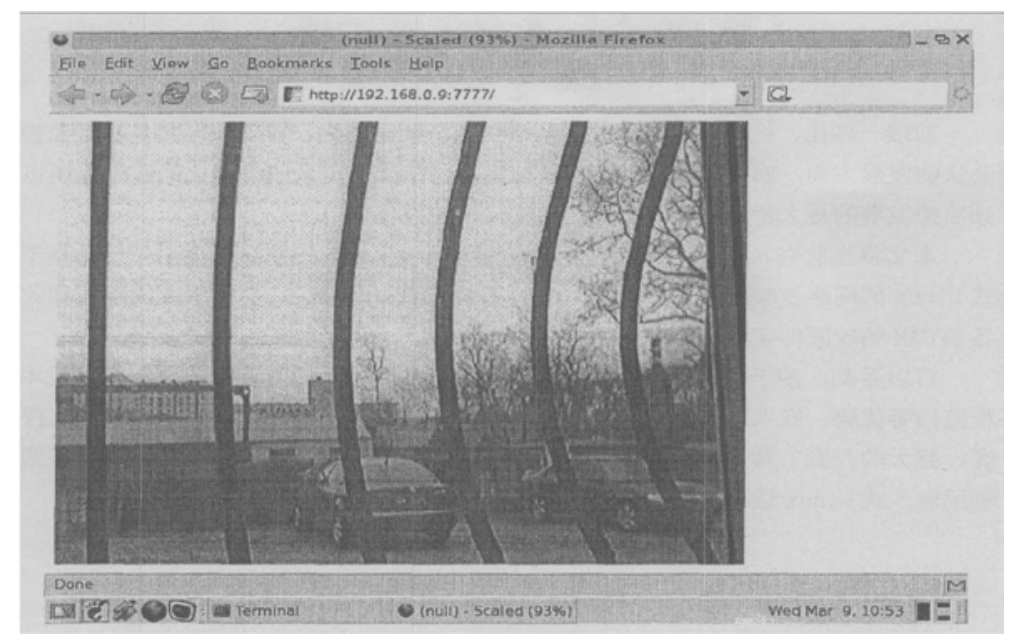

Fig. 4 Browse images

Browse images is shown in Fig. 4. The unified platform is based on ASP.NET technology and advanced development framework to build a network video surveillance platform. The Software design is using a combination of $\mathrm{B} / \mathrm{S}$ structure and the $\mathrm{C} / \mathrm{S}$ architecture design, which the background service module using the C/S architecture, user UI using the B/S structure. This platform is using streaming media management technology, data and control technology, low-bandwidth data transmission technology, massive data distribution, storage, processing, and analysis technology. This platform also can support mufti-stage deployment, It can build central control platform according to the different station side property and the management department, each platform can work independently and also can be set as super or platform and subordinate platform. The Provincial monitoring platform as superior platform can call to any station within the jurisdiction of side image information through the network.

\section{Conclusions}

This objective is to design, implement a set of real-time data collection, transmission, processing and information management an integrated application system. System adopts B/S architecture, computer network technology as the core, combined with digital video technology, multimedia communication and digital storage technologies, modular structure design, and media control through ASP.NET technology to integrate with the browser, direct access to Web browsing device to complete user interaction with the system, visit the area to monitor the unprecedented breadth.

\section{References}

[1] Luca Marchesotti, Lucio Marcenaro and Carlo Regazzoni, A video surveillance architecture for alarm generation and video sequences retrieval. IEEE, 2012: 892-895.

[2] Huihui Huang, Weirong Chen, Qingquan Qian and Liu Xiao, Implementation of wide area communication in distributed remote video monitoring system for substations. IEEE, 2012: 294-298.

[3] Lopes R J, Lindsay A T, David H. The utility of MPEG-7 systems in audio-visual applications with multiple streams.IEEE Transactions on Circuits and Systems for Video Technology, 2013, 13(1): 16-25.

[4] Information on http://msdn.microsoft.com/en-us/netframework/default.aspx

[5] P. Sarkar, K. Voruganti. IP Storage:The Challenges Ahead.In 19th IEEE Symposium on Mass Storage Systems, 2012, 5: 35-42. 